\title{
The challenge of immune control of immunodeficiency virus
}

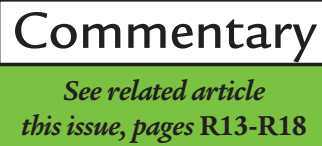

\author{
Douglas Richman
}

University of California-San Diego, Department of Pathology, La Jolla, California 92093, USA; and San Diego Veterans Affairs Medical Center, La Jolla, California 92161, USA

Address correspondence to: Douglas Richman, University of California-San Diego, Department of Pathology, Stein Clinical Research Building, Room 327, 9500 Gilman Drive, La Jolla, California 92093, USA. Phone: (858) 552-7439; Fax: (858) 552-7445;

E-mail: drichman@ucsd.edu.

When studies of the dynamics of HIV revealed rapid rates of viral clearance, hopes were raised that potent antiretroviral therapy might lead to cure of infection (1). The documentation of long-lived, latently infected CD4 lymphocytes dashed these hopes (2-4). Potent antiretroviral therapy has dramatically changed the morbidity and mortality of HIV disease in developed countries, but the prospect of the cost, inconvenience, and toxicity of lifelong suppressive chemotherapy is daunting. A second hope - the patient's own immunological defenses - has swept through the community of patients and health care providers. Does this represent a therapeutic opportunity or another mirage, easy to visualize by those with hope and desperation but impossible to grasp?

How can patients with established HIV infection - which infects and destroys CD4 lymphocytes ("the conductors of the immunologic orchestra"), with the usual result of fatal immunodeficiency - hope to be saved by their immune response to HIV? Several lines of evidence can be mustered to justify the pursuit of immunological strategies to contain HIV replication and disease.

Stronger immune responses correlate with less aggressive disease course. CD4 cell proliferative responses to HIV antigen, CD8 cytotoxic T-cell responses, and neutralizing antibody levels are all higher in long-term nonprogressors than in patients with progressive disease (5-10). This raises the chicken and egg question about causal relationships; however, the diminution of virus replication after acute infection is temporarily associated with the appearance of HIV-specific cytotoxic T lymphocyte (CTL) effector responses $(11,12)$. Perhaps more compelling are recent studies with SHIV- and SIV-infected rhesus macaques that support the contention that CD8 T cells, by mechanisms not yet elucidated, are required for control of HIV replication. With this model, the in vivo depletion of CD8 cells by monoclonal antibodies was temporally associated with significantly higher levels of viral replication (13-15). With the recovery of CD8 cells, the SIV levels diminished $(14,15)$.

The elicitation and maintenance of HIV-specific immunity, however, is not a trivial matter. CD4 memory cell response to HIV antigen is a suicidal act, converting the cell into a permissive target for the pathogen. Prevention of HIV replication protects CD4 cell depletion, and several groups have documented remarkable restoration of lymphocyte numbers and function with potent antiretroviral therapy. In the first months of treatment, large numbers of CD4/CD45RO (memory phenotype) cells appear in the circulation, largely by redistribution from the solid lymphoid tissue (16-19). Over the next several years, CD4/CD45RA (naive phenotype) cells are gradually regen erated, with peripheral expansion in almost all patients and with significant thymic reconstitution contributing in younger patients $(16,17,20)$. Restoration of cell numbers is accompanied by the recovery of detectable, functional immune responses to recall antigens (cytomegalovirus, mycobacteria, and others) that dramatically reduce the risk of opportunistic infection (16).

What is less clear is the degree of restoration of HIV-specific immunity. CD4 cell proliferative responses, which in one report are usually extinguished by ongoing HIV infection, appear to be preserved when potent antiretroviral therapy is administered in the first month or so after infection (7). CTL effector and memory T-cell responses persist with ongoing infection; however, these grad- ually diminish with progressive disease (8-10). Although recovery of lymphocyte numbers and function results from potent antiretroviral therapy, such therapy (which suppresses significant viral replication and, thus, exposure to large amounts of antigen) results in the rapid disappearance of functional immune CTL responses (21-23).

With the recent development of more sensitive and quantitative assays of Tcell immunity, apparently contradictory data have emerged. Despite the disappearance of functional CTLs from the peripheral circulation with potent antiviral chemotherapy, HIV antigen-specific CD8 cells persist, as measured by MHC class I/peptide epitope/tetramer reagents in FACS $^{\circledR}$ analysis (24). These apparently contradictory data do not indicate experimental error. Different assays are more likely measuring different lymphocyte populations with varying functions. If so, 3 fundamental questions arise. One, which T-cell functions are critical for the control of HIV replication? Two, which immunologic assays best measure the T-cell functions of interest? And three, which immunizing strategies elicit these desired functions? Regarding the last question, studies are in progress to examine HIV therapeutic vaccines and to pursue autoimmunization by withdrawal of antiretroviral therapy, as exemplified by the report of Ortiz et al. (25) in this issue of the JCI.

Answers to these questions are critical both for therapeutic strategies of immunologic control of HIV replication and for the development of effective HIV prophylactic vaccines. We know, both from extensive clinical experience and from a few, well-monitored clinical studies, that withdrawal of potent antiretroviral therapy routinely results in the rapid resumption of disseminated infection with impressive 
dynamics of replication (26-30). Are the anecdotes of delayed resumption and dampened viral dynamics presented by Ortiz et al. $(25)$ and others $(31,32)$ rare exceptions that prove the rule, or are they the first glimpses of opportunities to elicit and characterize the effective immune responses that will transform the management of HIV infection?

\section{Acknowledgments}

I thank Celsa Spina for the review of this commentary, which was supported by grants AI 27670, AI 38858, AI 43638, AI 36214 (to the UCSD Center for AIDS Research), and AI 29164, from the National Institutes of Health, and by the Research Center for AIDS and HIV Infection of the San Diego Veterans Affairs Healthcare System.

1. Perelson, A.S., et al. 1997. Decay characteristics of HIV-1-infected compartments during combination therapy. Nature. 387:188-191.

2. Wong, J.K., et al. 1997. Recovery of replicationcompetent HIV despite prolonged suppression of plasma viremia. Science. 278:1291-1294.

3. Finzi, D., et al. 1997. Identification of a reservoir for HIV-1 in patients on highly active antiretroviral therapy. Science. 278:1295-1300.

4. Chun, T.-W., et al. 1997. Presence of an inducible HIV-1 latent reservoir during highly active antiretroviral therapy. Proc. Natl. Acad. Sci. USA. 94:13193-13197.

5. Cao, Y., Qin, L., Zhang, L., Safrit, J., and Ho, D.D. 1995. Virologic and immunologic characterization of long-term survivors of human immunodeficiency virus type 1 infection. N. Engl. J. Med. 332:201-208.

6. Pantaleo, G., et al. 1995. Studies in subjects with long-term nonprogressive human immunodeficiency virus infection. $N$. Engl. J. Med. 332:209-216.

7. Rosenberg, E.S., et al. 1997. Vigorous HIV-1-specific $\mathrm{CD} 4+\mathrm{T}$ cell responses associated with con- trol of viremia. Science. 278:1447-1450.

8. Carmichael, A., Jin, X., Sissons, P., and Borysiewicz, L. 1993. Quantitative analysis of the human immunodeficiency virus type 1 (HIV-1)specific cytotoxic T lymphocyte (CTL) response at different stages of HIV-1 infection: differential CTL responses to HIV-1 and Epstein-Barr virus in late disease. J. Exp. Med. 177:249-256.

9. Klein, M.R., et al. 1995. Kinetics of Gag-specific cytotoxic $\mathrm{T}$ lymphocyte responses during the clinical course of HIV-1 infection: a longitudinal analysis of rapid progressors and long-term asymptomatics. J. Exp. Med. 181:1365-1372

10. Ogg, G.S., et al. 1998. Quantitation of HIV-1 specific cytotoxic $\mathrm{T}$ lymphocytes and plasma load of viral RNA. Science. 279:2103-2106.

11. Koup, R.A., et al. 1994. Temporal association of cellular immune responses with the initial control of viremia in primary human immunodeficiency virus type 1 syndrome. J. Virol. 68:4650-4655.

12. Borrow, P., Lewicki, H., Hahn, B.H., Shaw, G.M., and Oldstone, M.B. 1994. Virus-specific CD8+ cytotoxic T-lymphocyte activity associated with control of viremia in primary human immunodeficiency virus type 1 infection. J. Virol. 68:6103-6110.

13. Matano, T., et al. 1998. Administration of an antiCD8 monoclonal antibody interferes with the clearance of chimeric simian/human immunodeficiency virus during primary infections of rhesus macaques. J. Virol. 72:164-169.

14. Schmitz, J.E., et al. 1999. Control of viremia in simian immunodeficiency virus infection by CD8+ lymphocytes. Science. 283:857-860.

15. Jin, X., et al. 1999. Dramatic rise in plasma viremia after CD8+ $\mathrm{T}$ cell depletion in simian immunodeficiency virus-infected macaques. $J$. Exp. Med. 189:991-998.

16. Autran, B., et al. 1997. Positive effects of combined antiretroviral therapy on $\mathrm{CD}^{+} \mathrm{T}$ cell homeostasis and function in advanced HIV disease. Science. 277:112-116.

17. Pakker, N.G., et al. 1998. Biphasic kinetics of peripheral blood $\mathrm{T}$ cells after triple combination therapy in HIV-1 infection: a composite of redistribution and proliferation. Nat. Med. 4:208-214.

18. Zhang, Z.-Q., et al. 1998. Kinetics of CD4+ T cell repopulation of lymphoid tissues after treatment of HIV-1 infection. Proc. Natl. Acad. Sci. USA 95:1154-1159

19. Bucy, R.P., et al. 1999. Initial increase in blood
CD4 ${ }^{+}$lymphocytes after HIV antiretroviral therapy reflects redistribution from lymphoid tissues. J. Clin. Invest. 103:1391-1398.

20. McCune, J.M., et al. 1998. High prevalence of thymic tissue in adults with human immunodeficiency virus-1 infection. J. Clin. Invest. 101:2301-2308.

21. Morris, L., et al. 1998. HIV-1 antigen-specific and -nonspecific B cell responses are sensitive to combination antiretroviral therapy. J. Exp. Med. 188:233-245.

22. Ogg, G.S., et al. 1999. Decay kinetics of human immunodeficiency virus-specific effector cytotoxic $T$ lymphocytes after combination antiretroviral therapy. J. Virol. 73:797-800.

23. Gray, C.M., et al. 1999. Frequency of class I HLArestricted anti-HIV CD8 + T cells in individuals receiving highly active antiretroviral therapy (HAART). J. Immunol. 162:1780-1788.

24. Pitcher, C.J., et al. 1999. HIV-1-specific CD4 $4^{+} \mathrm{T}$ cells are detectable in most individuals with active HIV-1 infection, but decline with prolonged viral suppression. Nat. Med. 5:518-525.

25. Ortiz, G.M., et al. 1999. HIV-1-specific immune responses in subjects who temporarily contain virus replication after discontinuation of HAART. J. Clin. Invest. 104:R13-R18.

26. Daar, E.S., et al. 1998. Acute HIV syndrome after discontinuation of antiretroviral therapy in a patient treated before seroconversion. Ann. Intern. Med. 128:827-829.

27. Neumann, A.U., et al. 1999. HIV-1 rebound during interruption of highly active antiretroviral therapy has no deleterious effect on reinitiated treatment. AIDS. 13:677-683.

28. Harrigan, P.R., Whaley, M., and Montaner, J.S.G. 1999. Rate of HIV-1 RNA rebound upon stopping antiretroviral therapy. AIDS. 13:F59-F62.

29. Garcia, F., et al. 1999. Dynamics of viral load rebound and immunological changes after stopping effective antiretroviral therapy. AIDS 13:F79-F86.

30. Little, S., McLean, A., Spina, C., Richman, D., and Havlir, D. 1999. Viral dynamics of acute HIV-1 infection. J. Exp. Med. In press.

31. Vila, J., et al. 1997. Absence of viral rebound after treatment of HIV-infected patients with didanosine and hydroxycarbamide. Lancet. 350:635-636.

32. Lisziewicz, J., Jessen, H., Finzi, D., Siliciano, R.F., and Lori, F. 1998. HIV-1 suppression by early treatment with hydroxyurea, didanosine, and a protease inhibitor. Lancet. 352:199-200. 